\title{
Lagrangian analysis of transport in heterogeneous formations under transient flow conditions
}

\author{
Gedeon Dagan \\ Faculty of Engineering, Tel Aviv University, Tel Aviv, Israel
}

Alberto Bellin
Dipartamento di Ingegneria Civile ed Ambientale, Universita di Trento, Trent, Italy

Yoram Rubin

Department of Civil Engineering, University of California, Berkeley

\begin{abstract}
The coupled effects of porous media heterogeneity and flow unsteadiness on transport are investigated. We address the problem using a stochastic-Lagrangian approach. The log condictivity is modeled as a space random function; however, the flow unsteadiness is considered as deterministic, and it affects the magnitude and direction of the mean head gradient. Using a first-order solution in terms of the log conductivity variance, we developed a three-dimensional solution that includes the velocity covariances, the macrodispersion coefficients, and the displacement tensor, all as a function of the media heterogeneity and the unsteady mean flow parameters. The model is applied to the case of a periodic variation in the flow direction. We show that the effects of unsteadiness on the longitudinal spread are insignificant, while it affects strongly the transverse spread, in the form of an added constant dispersivity. Results obtained in previous studies are discussed and are shown to be particular cases of the present results.
\end{abstract}

\section{Introduction and Brief Discussion of Previous Work}

Transport of solutes in aquifer flow is a subject of great interest in various applications. Field measurements have indicated that the dispersion process is enhanced by the largescale heterogeneity, i.e., by the spatial variability of the hydraulic conductivity $K$, which is generally present in natural formations. It is customary to model $K(\mathbf{x})$ as a random space function, to account for its irregular variation in space and for the uncertainty affecting its distribution. Various models, analytical or numerical, have been developed in the past in order to relate the evolution of solute plumes to the heterogeneous structure. Most of the studies were carried out for conditions of steady and uniform average flow, the result of application of a constant head drop on the boundaries of the formation. In addition to simplicity, the justification was that under natural gradient conditions, flows are generally close to steady. Since the timescale of transients is generally of a seasonal nature, the assumption of steadiness is particularly appropriate for transport field tests of relatively short duration.

Analysis of field results in one of the most elaborate experiments carried out so far, at the Borden site [Sudicky, 1986], showed good agreement between linearized models, based on a first-order approximation in the log conductivity variance, and measurements for the longitudinal field-scale dispersion. In contrast, the rate of increase of the plume transverse moments was underpredicted by the linearized theory. A possible explanation for this effect was offered in the works of Neuman

Copyright 1996 by the American Geophysical Union.

Paper number 95WR02497.

0043-1397/96/95WR-02497\$05.00 and Zhang [1990] and of Zhang and Neuman [1990], who obtained a quasi-linear approximation of the transport equation based on Corrsin's conjecture. While the first-order theory predicts an asymptotically vanishing macrodispersion coefficient at great travel times, the Corrsin conjecture leads to a finite one. However, it was recently proved [Dagan, 1994] that a rigorous derivation of the nonlinear, quadratic in the $\log$ conductivity variance, term of the macrodispersivity tends to zero as well. This result strengthens confidence in the linear theory and precludes explaining the aforementioned discrepancy for Borden site, a formation of weak heterogeneity, by nonlinear effects.

In the search for a different explanation of this discrepancy, it was suggested [e.g., Naff et al., 1988] that the presence of small transients of flow around the mean may cause the enhanced transverse dispersion. Furthermore, a recent experiment in the same aquifer [Farell et al., 1994] has revealed that the time variation of flow, as manifested in head measurements in piezometers, was quite significant. This finding has stimulated a further examination of the impact of flow unsteadiness upon transport [Farell et al., 1994] in this particular experiment. Furthermore, the subject is of interest for prediction of plume evolution over extended periods of, say, tens or hundreds of years, for which transients are always present.

Although transport under unsteady flow conditions has received less attention, a few studies were carried out in the past, and we shall discuss them briefly here.

The problem was addressed by Kinzelbach and Ackerer [1986] and by Goode and Konikow [1990] in the conventional framework of solving the transport equation for a deterministic velocity field, while dispersion is represented by a tensor of constant coefficients. Goode and Konikow [1990] considered a flow which is uniform in space and varies periodically around a 
steady one. Thus the first example in which the mean temporal velocity is constant and unidirectional, while a constant fluctuation normal to it is applied periodically, is particularly instructive. In this example, the mean trajectory is a zigzag line of a small angle $+\theta$ and $-\theta$ around the horizontal axis. The main finding is that an observer inspecting the plume at the times its centroid crosses the mean flow path detects an apparent increase of the transverse dispersion coefficient. This increase is easy to grasp: When the flow suddenly changes direction, part of the longitudinal spread of the plume in the previous periods manifests itself as a transverse spread for axes parallel to the instantaneous velocity or to the mean temporal velocity. We shall not dwell here on the more involved cases of transients related to aquifer storativity analyzed by Goode and Konikow [1990]. A point of principle is that the actual dispersion coefficients, defined as half the rate of change of the plume spatial moments, are those of steady flow for an observer moving with the instantaneous flow velocity. In other words, the apparent increase of the transverse moments does not show up in the instantaneous dispersion coefficients.

In contrast with this approach, we consider here the case of a random velocity field whose impact upon transport cannot be encapsulated in a diffusive effect. As we shall show later, the latter is a particular case of a velocity field associated with a "Brownian motion," which may be a good approximation in cases in which the timescales of the mean flow are much larger than those associated with heterogeneity.

Another line of attack was adopted by Naff et al. [1988] and Rehfeldt and Gelhar [1992]. They considered heterogeneous formations of random stationary structure and a mean head gradient which is uniform in space, but fluctuating in time around a constant mean. Rehfeldt and Gelhar [1992] assumed that these fluctuations are random and small and neglect their interaction with velocity fluctuations associated with heterogeneity. In other words, the three additive components of transport are translation by uniform and steady flow, macrodispersion associated with heterogeneity under the same condition, and a temporal fluctuating flow in a homogeneous formation. Since only the last component is of concern here, we shall discuss it in some detail. Furthermore, we prefer to cast it in a Lagrangian rather than an Eulerian framework, since we find the first quite illuminating. Thus, with neglect of pore-scale dispersion, the fluctuation of the trajectory of a fluid or solute particle is given by

$$
\mathbf{X}^{\prime}(t)=\int_{0}^{t} \mathbf{u}\left(t^{\prime}\right) d t^{\prime}
$$

where $\mathbf{u}$ is the random velocity fluctuation, which is independent of $\mathbf{x}$ and results solely from the temporal head gradient variation. The trajectories covariance is therefore

$$
X_{i j}(t)=\int_{0}^{t} \int_{0}^{t} u_{y}\left(t^{\prime}, t^{\prime \prime}\right) d t^{\prime} d t^{\prime \prime} \quad(i, j=1,2,3)
$$

Rehfeldt and Gelhar [1992] assumed that the covariances $u_{t j}$ are stationary and of finite integral scales. Furthermore, their solution is equivalent to assuming that $X_{i j}$ represents the spatial moments of an ergodic plume. As a result, for a uniform mean flow in the $x_{1}$ direction, $D_{22}=(1 / 2) d X_{22} / d t$ tends to a constant, "Fickian" limit, for large $t$. This is in contrast with $D_{22}$ associated with steady flow and heterogeneity, which tends

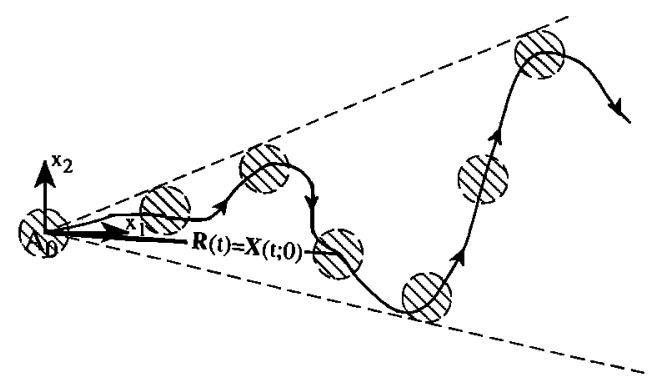

Figure 1. Schematic representation of the advective transport of a solute body in a realization of a velocity field uniform in space, but random in time.

to zero for large $t$ or is finite and very small if pore-scale dispersion is accounted for. Naff et al.'s [1988] analysis was similar, but $\mathbf{u}$ is regarded as deterministic, based on past measurements of the head gradient.

Our analysis differs in a few respects from that of Rehfeldt and Gelhar [1992] or of Naff et al. [1988]. Thus we define the dispersion coefficient as $D_{22}=(1 / 2) d\left(S_{22}\right\rangle / d t$, where $S_{22}=$ $\left(1 / A_{0}\right) \int_{A_{0}}\left(X_{2}-R_{2}\right)^{2} d \mathbf{a}$ is the plume spatial moment with respect to the centroid. Here $X_{2}=a_{2}+\int_{0}^{t} u_{2}\left(t^{\prime}\right) d t^{\prime}$ is the transverse coordinate of the trajectory of a particle originating at $\mathbf{x}=\mathbf{a}, R_{2}=\left(1 / A_{0}\right) \int_{A_{0}} X_{2} d \mathrm{a}$ is the centroid transverse trajectory, and $A_{0}$ is the input zone of a conservative solute of initial constant concentration. For ergodic plumes in steady flow through heterogeneous media, $d\left\langle S_{22}\right\rangle / d t$ is indeed equal to $d X_{22} / d t$ [see, e.g., Dagan, 1990]. However, this is not the case for a spatially uniform flow, as implied by (2). Indeed, in this case, in absence of pore-scale dispersion, the plume translates as a whole in each realization, and it is easy to see that $S_{22}(t)=S_{22}(0)=$ const and $D_{22}=0$. This result is explained in Figure 1, in which $A_{0}$ is a circle. The solute body wanders along a random path, but does not disperse, and $X_{\imath \jmath}$ in (2) is a measure of uncertainty, i.e., of the envelope of solute body trajectories. It can be regarded as a measure of dispersion of the mean concentration if measurements of concentration and spatial moments are defined as time averages over periods much larger than the timescale of fluctuations of the velocity. This was not the case in the aforementioned field experiments, since measurements were carried out over relatively short timescales and "instantaneous" pictures of the plume were obtained. We feel, therefore, that interpreting $X_{\imath}$ in (2) as spatial moments is not justified.

A second difference of principle is that we regard the temporal variation of the head gradient as deterministic. Indeed, in the Borden site field experiment, heads were monitored at short time intervals and their temporal changes during the transport experiment could be captured quite accurately [Farell et al., 1994]. In the case of prediction, uncertainty may affect the temporal fluctuations, though basically they are of a seasonal nature. However, we believe that this uncertainty should not be regarded as resulting in dispersion and the right context is that of time series and centroid motion (Figure 1). These topics are not discussed here.

Finally, the random velocity temporal variations were incorporated in the derivation of the mean concentration field by Kabala and Sposito [1991]. They used the Van Kampen cumulant expansion, and the velocity field was regarded as stationary in space and time. However, only a general formulation was presented, and no attempt was made to relate the temporal 
variations to those of the head and to the heterogeneous structure.

In the present study we employ the Lagrangian approach we have used in the past to investigate transport in steady flow. In our analysis the effects of unsteadiness and heterogeneity interact, in the spirit of the study of Goode and Konikow [1990].

\section{Statement of the Flow Problem and First-Order Solution}

Flow takes place in a heterogeneous formation. The log conductivity $Y(\mathbf{x})=\ln K$ is modeled as a stationary random space function of mean $m_{Y}=\langle Y\rangle$ and covariance $C_{Y}(\mathbf{r})=$ $\left\langle Y^{\prime}(\mathbf{x}) Y^{\prime}(\mathbf{y})\right\rangle$, where $Y^{\prime}=Y-m_{Y}, \mathbf{r}=\mathbf{x}-\mathbf{y}, \mathbf{x}\left(x_{1}, x_{2}, x_{3}\right)$ is a Cartesian coordinate, and angle brackets stand for ensemble averaging. The head $H(\mathbf{x}, t)=\langle H\rangle+h$ is assumed to have a given time dependent mean gradient

$$
\nabla\langle H\rangle=-\mathbf{J}(t)
$$

in line with previous works. Equation (3) implies that the instantaneous head gradient is constant over the zone covered by the plume, which may be an accurate assumption if the scale characterizing the spatial variation of $\nabla\langle H\rangle$ is sufficiently large [Farell et al., 1994]. We can set the problem in a rigorous mathematical framework by assuming the flow to be confined so that $H$ satisfies

$$
\begin{gathered}
\nabla^{2} H+\nabla Y \cdot \nabla H=0 \quad \mathrm{x} \in \mathbf{\Omega} \\
H=-\mathrm{J}(t) \cdot \mathbf{x} \quad \mathrm{x} \in \partial \Omega
\end{gathered}
$$

where $\Omega$ is the flow domain and $\partial \Omega$ is its boundary. Equations (3) and (4) yield for the head fluctuation $h(x, t)$

$$
\begin{gathered}
\nabla^{2} h+\nabla Y \cdot \nabla h=\mathbf{J} \cdot \nabla Y \quad \mathrm{x} \in \Omega \\
h=0 \quad \mathrm{x} \in \partial \Omega
\end{gathered}
$$

It is seen that the solution of (4) and (5) is identical with the solution for steady uniform flow provided that at each instant $\mathbf{J}$ assumes its time dependent value.

In line with previous work on steady flow, we adopt a firstorder approximation for $h$ by replacing (5) by

$$
\nabla^{2} h(\mathbf{x}, t)=\mathbf{J}(t) \cdot \nabla Y^{\prime}(\mathbf{x})
$$

and let the flow domain expand to infinity.

The velocity field is determined with the aid of Darcy's law at first order as follows [see, e.g., Dagan, 1984]:

$$
\begin{gathered}
\mathbf{V}(\mathbf{x}, t)=\mathbf{U}(t)+\mathbf{u}(\mathbf{x}, t) \\
\mathbf{U}=\langle\mathbf{V}\rangle=\frac{K_{G}}{n} \mathbf{J}(t) \quad \mathbf{u}(\mathbf{x}, t)=\frac{K_{G}}{n}\left[\mathbf{J}(t) Y^{\prime}(\mathbf{x})-\nabla h\right]
\end{gathered}
$$

where $K_{G}$ is the conductivity geometric mean and $n$ is the porosity, assumed to be constant.

To obtain the expressions of the velocity covariances, a prerequisite to solving the transport problem, we recall the procedure used in the past for steady flow [e.g., Dagan, 1984]. We observe that by the linearity of (6) we may write

$$
h(\mathbf{x}, t)=\mathbf{J}(t) \cdot \nabla \chi(\mathbf{x}) \quad \nabla^{2} \chi=Y^{\prime}(\mathbf{x})
$$

and the auxiliary random function $\chi$ depends only on the heterogeneous structure. By substituting (8) in (7) we obtain

$$
\begin{gathered}
u_{i}(\mathbf{x}, t)=\frac{K_{G}}{n}\left(J_{\imath} Y^{\prime}-J_{\alpha} \frac{\partial^{2} \chi}{\partial x_{t} \partial x_{\alpha}}\right)=U_{\alpha}(t) v_{l, \alpha}(\mathbf{x}) \\
v_{\imath, \alpha}=\delta_{l \alpha} Y^{\prime}-\frac{\partial^{2} X}{\partial x_{i} \partial x_{\alpha}}
\end{gathered}
$$

where $\delta_{t \alpha}$ is the Kronecker unit tensor and here and in the sequel we adopt the index summation convention.

Next, we multiply $u_{t}(\mathrm{x}, t)$ by $u_{j}\left(\mathrm{y}, t^{\prime}\right)$ and ensemble average. In the ensuing expression we encounter the covariances $C_{\chi Y}(\mathbf{r})=\left\langle\chi^{\prime}(\mathbf{x}) Y^{\prime}(\mathbf{y})\right\rangle=-P(\mathbf{r})$ and $C_{\chi}=\left\langle\chi^{\prime}(\mathbf{x}) \chi^{\prime}(\mathbf{y})\right\rangle=Q(\mathbf{r})$. The functions $P$ and $Q$, satisfying $\nabla^{2} P=-C_{Y}$ and $\nabla^{2} Q=P$, were used extensively in the past [Dagan, 1989; Rubin, 1990]. Their expressions for an exponential, isotropic $C_{Y}$ are recalled in the appendix. After these preparatory steps, the final general expressions of the velocity covariances are

$$
\begin{aligned}
& u_{i j}\left(\mathbf{x}, \mathbf{y}, t, t^{\prime}\right)=\left\langle u_{i}(\mathbf{x}, t) u_{j}\left(\mathbf{y}, t^{\prime}\right)\right\rangle \\
& =U_{\alpha}(t) U_{\beta}\left(t^{\prime}\right) v_{y, \alpha \beta}(\mathbf{r}) \quad \mathbf{r}=\mathbf{x}-\mathbf{y} \\
& v_{y, \alpha \beta}(\mathbf{r})=\left\langle v_{t, \alpha}(\mathbf{x}) v_{,, \beta}(\mathbf{y})\right\rangle=\delta_{l \alpha} \boldsymbol{\delta}_{j \beta} C_{Y}(\mathbf{r})+\delta_{j \beta} \frac{\partial^{2} P}{\partial r_{t} \partial r_{\alpha}} \\
& +\delta_{l a} \frac{\partial^{2} P}{\partial r_{j} \partial r_{\beta}}-\frac{\partial^{4} Q}{\partial r_{\imath} \partial r_{\alpha} \partial r_{j} \partial r_{\beta}}
\end{aligned}
$$

In words, the tensor $v_{\imath \jmath, \alpha \beta}(\mathbf{x}, \mathbf{y})(i, j, \alpha, \beta=1,2,3)$ yields the velocity covariance tensor for an arbitrary mean velocity vector $U$. The unsteadiness of the motion is evident in the dependence of the latter on time.

In the past we have considered steady flows parallel to the $x_{1}$ axis, i.e., $\mathbf{U}(U, 0,0)$ for which

$$
\begin{aligned}
U_{l j}= & v_{t, 11}=\delta_{t 1} \delta_{j 1} C_{Y}(\mathbf{r})+\delta_{j 1} \frac{\partial^{2} P}{\partial r_{t} \partial r_{1}} \\
& +\delta_{t 1} \frac{\partial^{2} P}{\partial r_{j} \partial r_{1}}-\frac{\partial^{4} Q}{\partial r_{t} \partial r_{J} \partial r_{1}^{2}}
\end{aligned}
$$

The explicit expressions of $U_{\imath \jmath}$ (11) are given by Rubin [1990] for two-dimensional flow and isotropic exponential $C_{Y}$, by Zhang and Neuman [1992] for three dimensions, and by Rubin and Dagan [1992] for anisotropic $C_{Y}$. As a matter of fact, because of many symmetries, the components of $v_{i j, \alpha \beta}$ can be expressed solely with the aid of $U_{i j}$ (see the appendix).

\section{Statement of the Transport Problem and First-Order Solution}

A plume of a conservative solute is injected at $t=0$ in a volume (area) $A_{0}$, i.e., $C(\mathbf{x}, 0)=C_{0}$ for $\mathbf{x}=\mathbf{a} \in A_{0}$ and $C=0$ otherwise. We neglect the effect of pore-scale dispersion, so that the trajectory $\mathbf{x}=\mathbf{X}(t ; \mathbf{a})$ of a particle originating at $\mathbf{x}=\mathbf{a}, t=0$ satisfies

$$
\frac{d \mathbf{X}(t ; \mathbf{a})}{d t}=\mathbf{V}(\mathbf{X}, t) \quad \mathbf{X}(0 ; \mathbf{a})=\mathbf{a}
$$

By substituting (7) in (12) and integrating, we get for the mean $\langle\mathbf{X}\rangle$ and fluctuation $\mathbf{X}^{\prime}$ the following expressions 


$$
\begin{gathered}
\langle\mathbf{X}(t ; \mathbf{a})\rangle=\mathbf{a}+\int_{0}^{t} U\left(t^{\prime}\right) d t^{\prime} \\
\mathbf{X}^{\prime}(t ; \mathbf{a})=\int_{0}^{t} \mathbf{u}\left[\mathbf{X}\left(t^{\prime}\right), t^{\prime}\right] d t^{\prime}
\end{gathered}
$$

The spatial moments of the plume, i.e., the centroid coordinate $\mathbf{R}=\left(1 / A_{0}\right) \int_{A_{0}} \mathbf{X} d \mathbf{a}$ and the second moments $S_{\imath \jmath}=$ $\left(1 / A_{0}\right) \int_{A_{0}}\left(X_{\imath}-R_{\imath}\right)\left(X_{J}-R_{J}\right) d$ a with respect to the centroid, are given in terms of trajectories [see, e.g., Dagan, 1989] by

$$
\begin{gathered}
\mathbf{R}(t)=\langle\mathbf{X}(t ; 0)\rangle+\frac{1}{A_{0}} \int_{A_{0}} \mathbf{X}^{\prime}(t ; \mathbf{a}) d \mathbf{a} \\
S_{v j}(t)=S_{t j}(0)+X_{t j}(t)-R_{u j}(t)
\end{gathered}
$$

where $X_{\imath j}=\left\langle X_{i}^{\prime}(t ; \mathbf{a}) X_{j}^{\prime}(t ; \mathbf{a})\right\rangle$ is the trajectory covariance and $R_{i j}$ is the $\mathbf{R}$ covariance. We assume that the plume is ergodic, i.e., its transverse extent is sufficiently large compared to the $Y$ correlation scale [Dagan, 1990] to warrant taking $R_{i j} \approx 0$ in (14). Hence

$$
\begin{gathered}
\mathbf{R} \approx\langle\mathbf{R}\rangle=\langle\mathbf{X}(t ; 0)\rangle \\
S_{v}(t) \approx\left\langle S_{v}(t)\right\rangle=S_{\imath \jmath}(0)+X_{\imath \jmath}(t) \\
D_{v j}(t)=\frac{1}{2} \frac{d\left\langle S_{\imath \jmath}\right\rangle}{d t}=\frac{1}{2} \frac{d X_{\imath}}{d t}
\end{gathered}
$$

where $D_{\imath}$ is the effective dispersion tensor.

To derive $X_{i j}$ in a simple and consistent manner with the first-order approximation adopted for the velocity field, we follow the procedure adopted in the past [Dagan, 1989] and replace $\mathbf{X}$ in (13) by its mean, i.e.,

$$
\begin{gathered}
\mathbf{X}^{\prime}(t)=\int_{0}^{t} \mathrm{u}\left[\left\langle\mathbf{X}\left(t^{\prime}\right)\right\rangle, t^{\prime}\right] d t^{\prime} \\
X_{u}(t)=\int_{0}^{t} \int_{0}^{t} u_{y}\left[\left\langle\mathbf{X}\left(t^{\prime}\right)\right\rangle,\left\langle\mathbf{X}\left(t^{\prime \prime}\right)\right\rangle, t^{\prime}, t^{\prime \prime}\right] d t^{\prime} d t^{\prime \prime}
\end{gathered}
$$

Finally, we substitute $u_{i j}(10)$ in (16), to obtain $X_{i j}$ and $D_{\imath j}$ explicitly in terms of the velocity covariances as follows:

$$
\begin{aligned}
& X_{t \jmath}(t)=\int_{0}^{t} \int_{0}^{t} U_{\alpha}\left(t^{\prime}\right) U_{\beta}\left(t^{\prime \prime}\right) v_{\imath, \alpha \beta}\left[\left\langle\mathbf{X}\left(t^{\prime}\right)\right\rangle-\left\langle\mathbf{X}\left(t^{\prime \prime}\right)\right\rangle\right] d t^{\prime} d t^{\prime \prime} \\
& D_{\imath \jmath}(t)=\frac{1}{2} \frac{d X_{t j}}{d t} \\
& =\frac{1}{2} \int_{0}^{t}\left[U_{\alpha}(t) U_{\beta}\left(t^{\prime}\right)+U_{\alpha}\left(t^{\prime}\right) U_{\beta}(t)\right] v_{v, \alpha \beta}\left[\langle\mathbf{X}(t)\rangle-\left\langle\mathbf{X}\left(t^{\prime}\right)\right\rangle\right] d t^{\prime}
\end{aligned}
$$

Equations (17) encapsulate the main result of the last two sections. They express the plume second spatial moment and dispersion coefficients as functions of the unsteady mean velocity $\mathrm{U}(t)$ on one hand, and the heterogeneous structure through the functions $P$ and $Q$ on which $v_{\imath, \alpha \beta}(\mathbf{r})$ in (11)

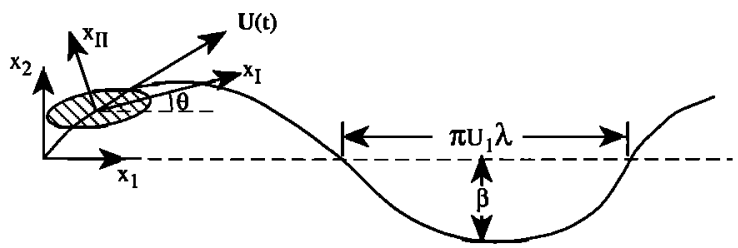

Figure 2. Definition sketch for transport by a periodic mean flow (equation (18)).

depend, on the other. Equations (17) will be employed in the following sections for analyzing periodic flows.

We wish to show that previous results obtained in the literature are particular cases of (17). Indeed, the steady flow solutions we developed in the past correspond to $\mathbf{U}$ constant and $\left\langle\mathbf{X}\left(t^{\prime}\right)\right\rangle-\left\langle\mathbf{X}\left(t^{\prime \prime}\right)\right\rangle=\mathbf{U}\left(t^{\prime}-t^{\prime \prime}\right)$. The solution of Goode and Konikow [1990] results from (17) for $v_{t, \alpha \beta}=d_{t \jmath, \alpha \beta} \delta\left(t^{\prime}-t^{\prime \prime}\right)$, where $d_{i j, \alpha \beta}$ is a tensor of constant components. Finally, the approximation of Rehfeldt and Gelhar [1992] for the unsteady term is equivalent to taking $v_{t \jmath, \alpha \beta}=\delta_{i \alpha} \delta_{j \beta} \rho_{\alpha \beta}\left(t^{\prime}-t^{\prime \prime}\right)$, where $\rho_{\alpha \beta}$ is the velocity temporal autocorrelation.

We proceed with illustrating our approach by applying (17) to a simple case of unsteady mean flow.

\section{Application to Simple Periodic Flows}

Similarly to previous studies, we consider a flow which fluctuates periodically around a steady, uniform one. The most general representation of $\mathrm{U}(t)$ is by a Fourier series [Farell et $a l ., 1994]$ whose basic period is 1 year. To grasp the effect of unsteadiness in a simple manner we consider here a single harmonic and defer a more general representation to a future study. Thus the flow is of constant mean $U_{1}$ in the $x_{1}$ direction and fluctuates in the $x_{2}$ direction (Figure 2). We adopt here and subsequently variables that are made dimensionless with respect to $I_{Y}$, the $\log$ conductivity integral scale, and $I_{Y} / U_{1}$ as length and timescales, respectively. Hence

$$
\begin{aligned}
U_{1}=1 & U_{2}=\frac{\beta}{\lambda} \cos (t / \lambda) & U_{3}=0 \\
\left\langle X_{1}\right\rangle=t & \left\langle X_{2}\right\rangle=\beta \sin (t / \lambda) & \left\langle X_{3}\right\rangle=0
\end{aligned}
$$

The two parameters characterizing unsteadiness are $\beta$, the dimensionless amplitude of the mean trajectory, and $\lambda=$ $T / 2 \pi$, where $T$ is the dimensionless period (Figure 2). For $\beta=$ 0 we recover the case of steady flow of uniform velocity $U_{1}$ analyzed extensively in previous studies. We shall denote the covariances pertinent to $\beta=0$ by $X_{t j}^{(s t)}(t)$ and correspondingly $D_{i j}^{(s t)}(t)=(1 / 2) d X_{i j}^{(s t)} / d t$.

The trajectory covariance in (17) becomes now, by substitution of (18),

$$
\begin{gathered}
X_{u j}(t)=\int_{0}^{t} \int_{0}^{t}\left\{v_{l y, 11}(\mathbf{r})+\left[U_{2}\left(t^{\prime}\right)+U_{2}\left(t^{\prime \prime}\right)\right] v_{l, 12}(\mathbf{r})\right. \\
\left.+U_{2}\left(t^{\prime}\right) U_{2}\left(t^{\prime \prime}\right) v_{\imath, 22}(\mathbf{r})\right\} d t^{\prime} d t^{\prime \prime} \\
r_{1}=t^{\prime}-t^{\prime \prime} \\
r_{2}=\beta\left[\sin \left(t^{\prime} / \lambda\right)-\sin \left(t^{\prime \prime} / \lambda\right)\right] \\
=2 \beta \sin \left[\left(t^{\prime}-t^{\prime \prime}\right) / 2 \lambda\right] \cos \left[\left(t^{\prime}+t^{\prime \prime}\right) / 2 \lambda\right] \\
r_{3}=0
\end{gathered}
$$


When treating two-dimensional flows we have to suppress in (19) the dependence on $r_{3}$.

To carry out the calculations in (19), it is beneficial to switch to the variables $t^{\prime}=(\nu+\mu) / 2$ and $t^{\prime \prime}=(\nu-\mu) / 2$, leading to the final expression

$$
\begin{aligned}
X_{\imath \jmath}(t) & =\int_{0}^{t} \int_{\mu}^{2 t-\mu}\left\{v_{l, 11}(\mathbf{r})+2(\beta / \lambda) \cos (\mu / 2 \lambda)\right. \\
\cdot & \cos (\nu / 2 \lambda) v_{l j, 12}(\mathbf{r})+\frac{1}{2}(\beta / \lambda)^{2} \\
\cdot & {\left.[\cos (\mu / \lambda)+\cos (\nu / \lambda)] v_{l, 22}(\mathbf{r})\right\} d \nu d \mu }
\end{aligned}
$$

with $r_{1}=\mu, r_{2}=2 \beta \sin (\mu / 2 \lambda) \cos (\nu / 2 \lambda)$, and $r_{3}=0$. The macrodispersion coefficients result from the differentiation of (20), leaving only the first integral and replacing $\nu=$ $2 t-\mu$ in the integrand.

Summarizing, $X_{i j}(20)$ and the associated $D_{\imath}$ require carrying out a double or a simple quadrature, respectively, for given parameters values and given $C_{Y}$. The results of such computations are given in the next section.

\section{A Few Numerical Results}

In order to grasp the implications of the developments of the previous section, we have carried out detailed computations for a few values of the parameters of interest. We have selected the exponential and isotropic $C_{Y}=\sigma_{Y}^{2} \exp \left(-r / I_{Y}\right)$, and at first order the velocity and trajectories covariances are linear in $\sigma_{Y}^{2}$. The basic functions $P$ and $Q$ for this $C_{Y}$ were derived in the past and we give here in the appendix their analytical expressions for both two- and three-dimensional flows.

First, we have considered two-dimensional flows, pertinent to vertically averaged velocity and concentration or to regional flows. With the velocity and mean trajectory given by (18), while suppressing $x_{3}$, we have selected first the value $\lambda=1$, i.e., $T U_{1} / I_{Y}=2 \pi$. For $I_{Y}$ of the order of meters and $U_{1}$ of the order of centimeters per day, $T$ is of the order of years. As for the only remaining parameter, the dimensionless amplitude $\beta$, we have selected the three values $\beta=0.1,0.2$, and 0.3 .

The computations are straightforward: (1) by differentiating $P$ and $Q$ (equation (A2)) we have derived the three coefficients $U_{i j}(11)$ for $i, j=1,2$, and subsequently the coefficients $v_{t j, \alpha \beta}$ (10), which are simply related to $U_{i j}$ (see the appendix, equations (A4)). Subsequently, we have computed $X_{i j}$ (20) by two numerical quadratures and $D_{i j}(17)$ by one.

In Figure 3 we represent the dependence of $X_{\imath j}(t ; \beta=0.2)$ as well as that of $X_{i j}^{(s t)}$ on travel time (or travel distance along the $x_{1}$ axis). The steady state values are those obtained in the past [Dagan, 1984]. The results are qualitatively the same for other values of $\beta$.

It is seen that the longitudinal $X_{11}(t)$ (Figure 3a) is very close to $X_{11}^{(s t)}(t)$, though some small fluctuations are present in the former. $X_{12}(t)$ (Figure $3 \mathrm{~b}$ ) is an oscillatory component of period $T=2 \pi \lambda$ and of small amplitude. It also has a small drift related to the initial conditions. Last, $X_{22}(t)$ (Figure 3c) is definitely larger than $X_{22}^{(s t)}(t)$, and we shall discuss its behavior subsequently.

With the aid of $X_{\iota j}$ we determined the principal values, the longitudinal $X_{I, I}$ and the transverse $X_{I I, I I}$ of the tensor $X_{\imath \jmath}$, as well as the angle $\theta$ between the longitudinal axis $x_{1}$ and $x_{1}$, the direction of the mean flow (Figure 2).

To illustrate the results, we present in Figure 4 the depen-
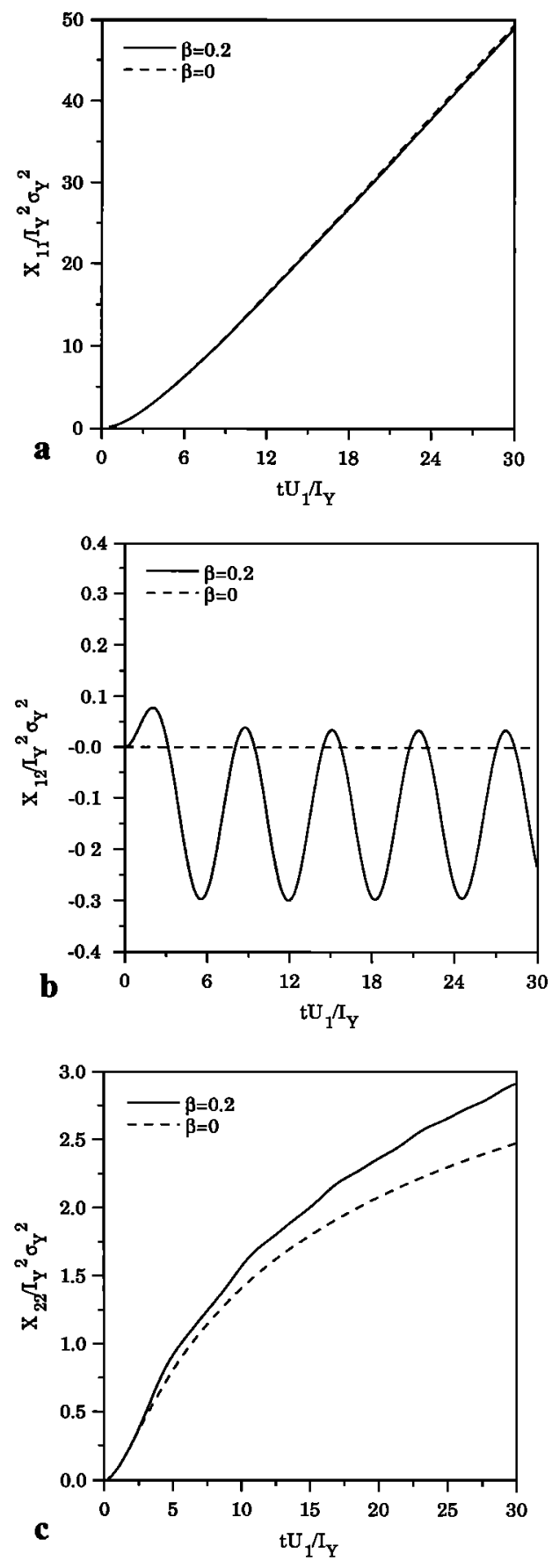

Figure 3. Two-dimensional flow: (a) the unsteady longitudinal trajectory covariance $X_{11}$ (equation (20)) for $\lambda=1, \beta=0.2$ and $X_{11}^{(s t)}(\beta=0)$; (b) same as Figure 3a for $X_{12}$; and (c) same as Figure $3 a$ for $X_{22}$.

dence of $\theta$ upon the dimensionless travel time (or distance along $\left.x_{1}\right)$, as well as that of the angle $\tan ^{-1}\left(U_{2} / U_{1}\right)=\tan ^{-1}$ $[\beta / \lambda \cos (t / \lambda)]$ between the tangent to the mean centroid trajectory and $x_{1}$ (Figure 2). The behavior is similar for other values of $\beta$, and the discussion is deferred to the next section.

The longitudinal component $X_{\mathrm{I}, \mathrm{I}}(t)$ is very close to $X_{11}(t)$ and is not shown. In Figure 5 we give the transverse $X_{\text {II,II }}$ as a function of time for $\beta=0.1,0.2$, and 0.3 as well as $X_{22}^{(s t)}(t)$. It is emphasized that $X_{22}$ (Figure 3c) for the same values of $\beta$ is not distinguishable from $X_{\mathrm{II}, \mathrm{II}}$. 


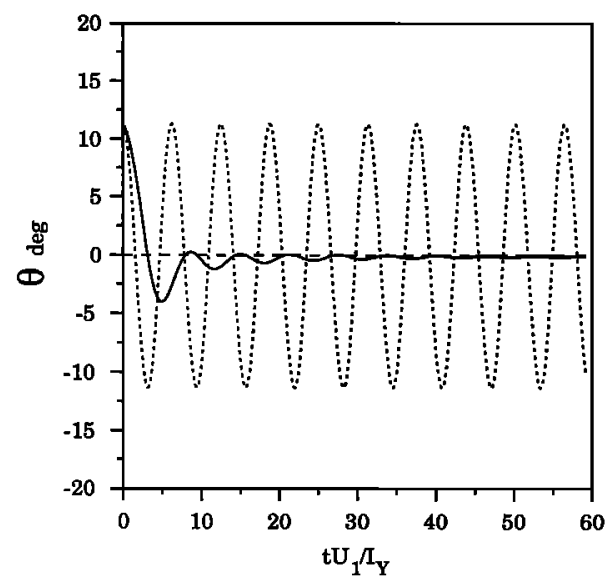

Figure 4. Two-dimensional flow: the angle $\theta$ between the longitudinal mean direction of $X_{i j}$ and the $x_{1}$ axis (solid line) and the angle between the mean trajectory (equation (18)) and $x_{1}$ (dotted line).

As is apparent from Figures 3-5, the main impact of the presence of the unsteady harmonic component is upon $X_{22}(t ; \beta) \approx$ $X_{\text {II,II }}$, which is larger than $X_{22}^{(s r)}$. To grasp the nature of the incremental effect of unsteadiness, we have represented in Figure 6 the difference $X_{22}-X_{22}^{(s t)}$ as a function of time. It is seen that this difference has a linear trend with slight oscillations around it. A linear regression has led to the following values for half the slope $\Delta \alpha_{T}=(1 / 2)\left[X_{\mathrm{II}, \mathrm{II}}(t)-X_{22}^{(s t)}(t)\right] / t$

$$
\begin{gathered}
\Delta \alpha_{T}=0.0018(\beta=0.1, \lambda=1) \\
\Delta \alpha_{T}=0.0065(\beta=0.2, \lambda=1) \\
\Delta \alpha_{T}=0.016(\beta=0.3, \lambda=1) \\
\Delta \alpha_{T}=0.0014(\beta=0.3, \lambda=5)
\end{gathered}
$$

We have supplemented the results for $\lambda=1$ in (21) by an additional one for a much larger period of $\lambda=5, T=10 \pi$. It is seen that $\Delta \alpha_{T}$ increases with $\beta$, roughly like $\beta^{2}$, and diminishes with increasing $\lambda$.

In Figure 7 we represent the dependence on time of the various $D_{\imath}$ as well as of $D_{i j}^{(s t)}(t)$ for the largest $\beta=0.3$ (the behavior is similar for other $\beta$ ). Similarly to $X_{11}$ (Figure 3a),

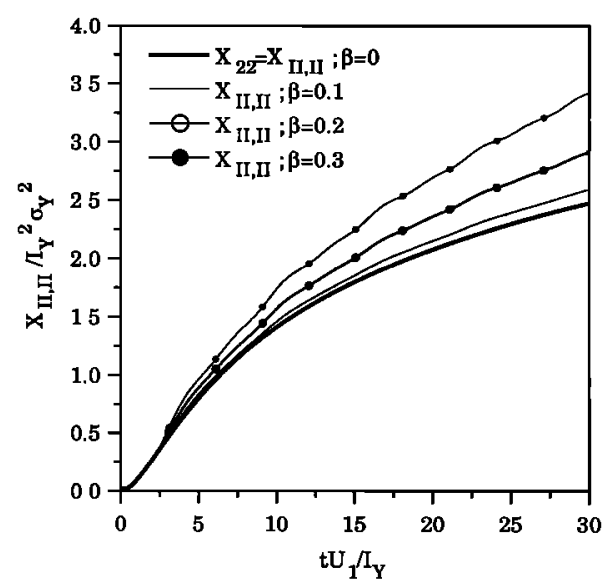

Figure 5. Two-dimensional flow: the transverse trajectory variance $X_{\mathrm{II}, \mathrm{II}}$ (see Figure 2) for $\beta>0$ and $X_{22}^{(s t)}(\beta=0)$.

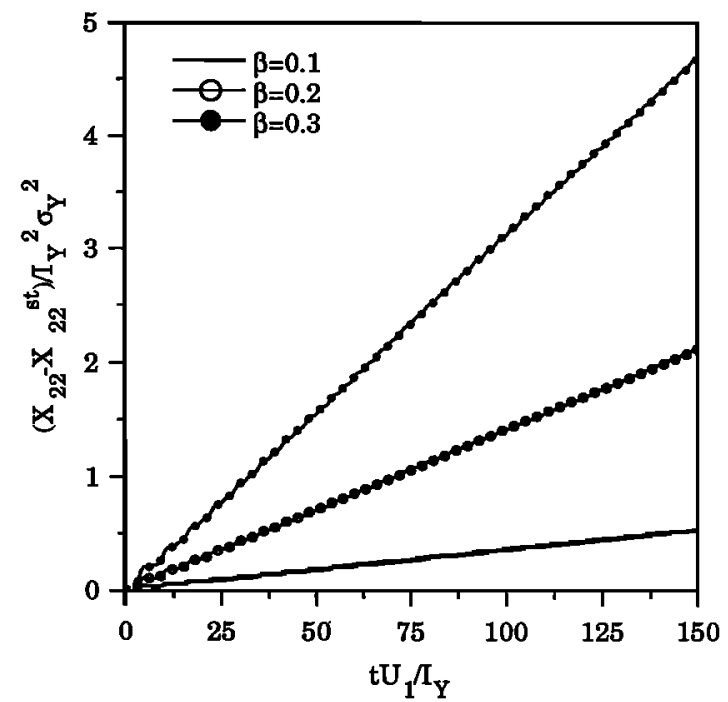

Figure 6. Two-dimensional flow: the difference between $X_{22}$ (equation (20)) and $X_{22}^{(s t)}$.

the longitudinal macrodispersion coefficient $D_{11}$ (Figure 7a) is very close to $D_{11}^{(s t)}$, except for small periodic fluctuations. The mixed component $D_{12}$ (Figure $7 \mathrm{~b}$ ), resulting from the differentiation of $X_{12}$ (Figure 3b), is purely oscillatory and of small amplitude. The most significant component is $D_{22}$ (Figure 7c), which is larger than $D_{22}^{(s t)}$ and displays an oscillatory as well as a constant increment, to be discussed in the next section.

Our next set of computations were for three-dimensional transport, again for an exponential isotropic $C_{Y}$ and for the same unsteady mean flow of $(18)$ in the horizontal plane $x_{1}, x_{2}$. This time the tensors $X_{v}$ and $D_{\imath}$ have nine components each. They were computed by the same procedure as for twodimensional flows, except that $P$ and $Q$ are given by (A3). We have carried out computations for the same parameter values, i.e., $\lambda=1$ and $\beta$ equal to $0.1,0.2$, and 0.3 . Many of the results parallel those of two-dimensional flow and are recapitulated in words only. Thus the longitudinal principal value $X_{\mathrm{I}, \mathrm{I}}(t)$ is very close to $X_{11}(t)$ for the range of parameters we chose and the same is true for $X_{\text {III,III }}(t) \approx X_{22}^{(s t)}(t)=X_{33}^{(s t)}(t)$. The latter has been derived previously [Dagan, 1984] and is also represented here in Figure 8 . The behavior of $\theta$, the angle between the longitudinal principal axis of $X_{t}$ and the mean flow direction $x_{1}$ (Figure 2) is very similar to that of Figure 4: At $t=0$ it is the same as that of the mean velocity, and it tends quickly to zero after a few cycles. The main impact of unsteadiness is felt again in the horizontal transverse direction $x_{2}$. This is illustrated in Figure 8, which displays the dependence of $X_{\mathrm{II,I}}$ and of $X_{22}^{(s t)}$ upon travel time (distance along $x_{1}$ ) for all values of $\beta$.

The increment $X_{22}(t)-X_{22}^{(s t)}(t)$ can be approximated accurately by a linear trend of slope $2 \Delta \alpha_{T}$, similarly to the two-dimensional case (Figure 6). The half slopes have the following values:

$$
\begin{gathered}
\Delta \alpha_{T}=0.0025(\beta=0.1, \lambda=1) \\
\Delta \alpha_{T}=0.01(\beta=0.2, \lambda=1) \\
\Delta \alpha_{T}=0.022(\beta=0.3, \lambda=1)
\end{gathered}
$$

which are close to, though somewhat larger than, those of (21) for two-dimensional flow and transport. 

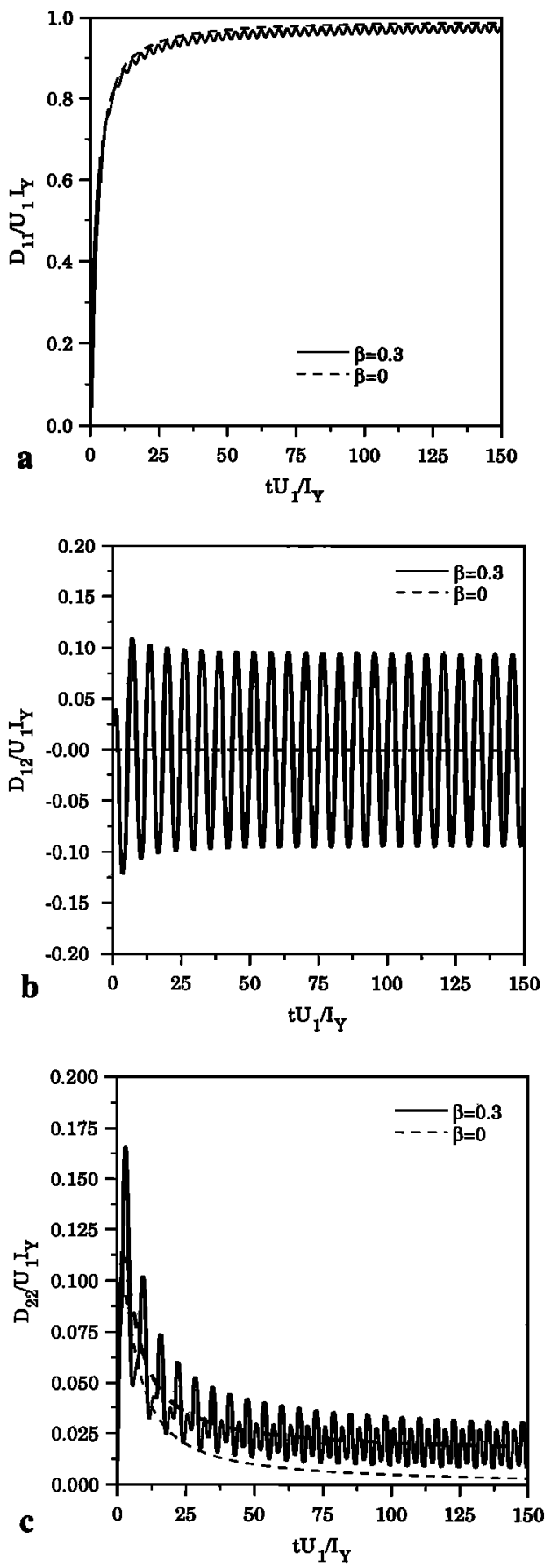

Figure 7. Two-dimensional flow: (a) the unsteady longitudinal macrodispersion coefficient $D_{11}=(1 / 2) d X_{11} / d t$ (equation (20)) for $\lambda=1, \beta=0.3$ and $D_{11}^{(s t)}(\beta=0)$; (b) same as Figure 7a for $D_{12}$; and (c) same as Figure 7a for $D_{22}$ supplemented by the approximation (26) (thick-dashed line).

Finally, in Figure 9 we have represented the dependence of $D_{22}$ upon time for $\beta=0.3$ as well as the steady $D_{22}^{(s t)}(\beta=0)$. The other components, $D_{11}$ and $D_{12}$, are very similar in behavior to those pertaining to two-dimensional flow (Figures $7 \mathrm{a}$ and $7 \mathrm{~b}$ ).

As a last point, one can define the transverse second spatial moment of the plume with respect to the axis $x_{1}$, rather than to the centroid of coordinate $\left\langle R_{2}\right\rangle=\beta \sin (t / \lambda)$. This results in the extra term $\left\langle R_{2}(t)\right\rangle^{2}=\beta^{2} \sin ^{2}(t / \lambda)$ supplementing $X_{22}$ of Figure 5 . This term is essentially the one considered by Naff et

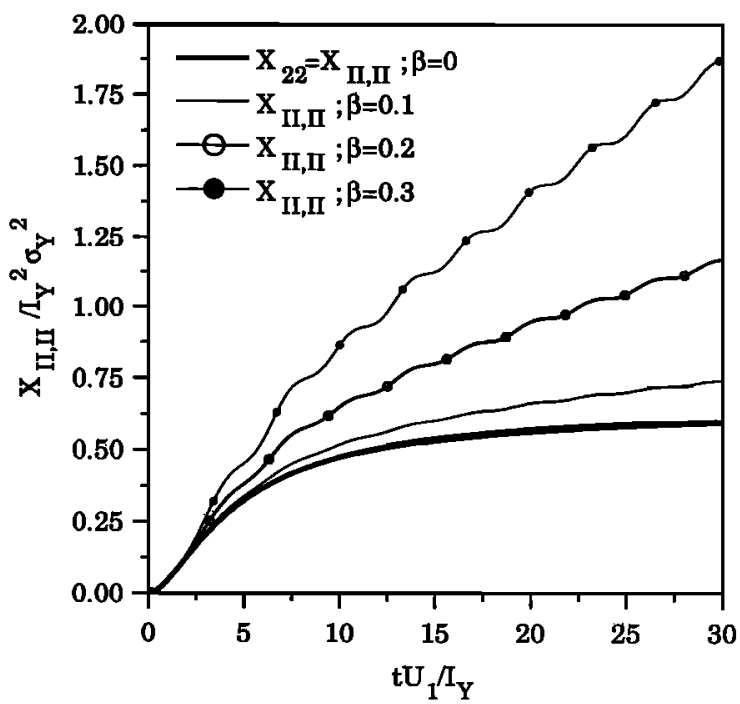

Figure 8. Three-dimensional flow: the transverse trajectory variance $X_{\mathrm{II}, \mathrm{II}}$ for $\lambda=1, \beta>0$ and $X_{22}^{(s t)}(\beta=0)$.

al. [1988], and it yields a small oscillatory contribution to $S_{22}$ and $D_{22}$. We shall adhere here to the definition of $S_{22}$ with respect to the instantaneous centroid, though the differences between the two definitions are seen to be quite small.

We proceed now with discussing the numerical results.

\section{Discussion of Numerical Results}

We have examined in sections 4 and 5 the interaction between a simple unsteady flow, of constant mean horizontal velocity and periodic transverse one, and the heterogeneous structure. Our main simplifying assumptions were neglecting pore-scale dispersion and adopting a first-order approximation in $\sigma_{Y}^{2}$ for flow and transport. It is emphasized that the results for velocity and trajectory covariances and for macrodispersion coefficients are consistent leading-order terms in expansions in powers of $\sigma_{Y}^{2}$.

While we have kept the period constant (except in (21)), we have examined a range of values of the amplitudes $\beta$ (made

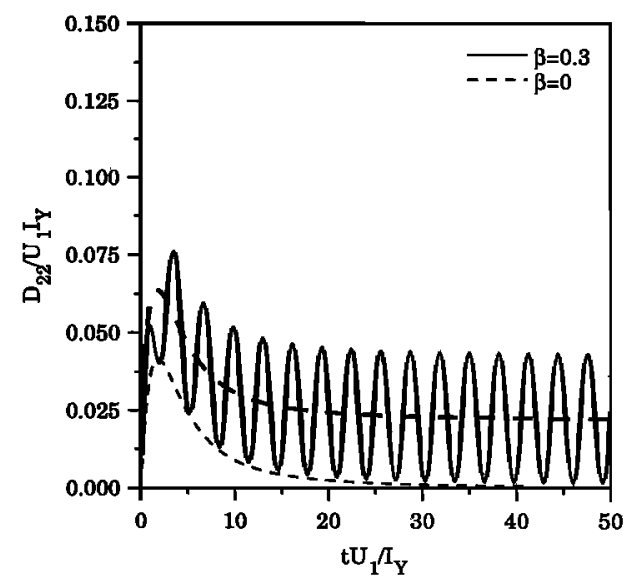

Figure 9. Three-dimensional flow: the unsteady transverse macrodispersion coefficient $D_{22}=(1 / 2) d X_{22} / d t$ (equation (20)) for $\lambda=1, \beta=0.3, D_{22}^{(s t)}(\beta=0)$ and the approximation (26) (thick-dashed line). 
dimensionless with respect to $I_{Y}$ ) up to 0.3 , which represents quite a large deviation of the mean trajectory (18) from the mean (temporal) one.

A first result, consistent with both field findings and previous works, is that the longitudinal (as well as the vertical for threedimensional flow) trajectory variances $X_{11}$ (and $X_{33}$ ) and the associated macrodispersion coefficients $D_{11}$ (and $D_{33}$ ) are little influenced by unsteadiness (Figures 3 and 4). The cross component $X_{12}$ (Figure 3) is oscillatory, and it impacts mainly the angle $\theta$ between the longitudinal main axis of the $X_{\iota}$ tensor and $x_{1}$. At $t=0$ this angle is identical with that between the instantaneous mean velocity and the $x_{1}$ axis (Figure 4). This is expected, since the effect of heterogeneity is not felt then and the plume translates with the mean velocity. The angle $\theta$ tends, however, quickly to zero, and the plume becomes practically parallel with the $x_{1}$ axis (Figure 4). This is mainly due to the quick increase of $X_{11}$ with $t$ as compared with $X_{12}$.

The main effect of the unsteady flow component is in increasing the transverse trajectory variance $X_{\mathrm{II}, \mathrm{II}}(t) \approx X_{22}(t)$ and the associated effective dispersion coefficient $D_{22}(t)$ (Figures 5-9) with respect to the steady ones $X_{22}^{(s t)}(t)$ and $D_{22}^{(s t)}(t)$. The effect is more dramatic in three-dimensional flows (Figure 8 ), for which $X_{22}^{(s t)}(t)$ tends to a constant value, whereas the unsteady component has a linear trend. The rest of our discussion is devoted to this effect.

First, we shall recall in an explicit manner the relationships leading to $X_{22}$ (see (20) and (A4))

$$
\begin{aligned}
X_{22}(t ; \beta, \lambda)=\int_{0}^{t} \int_{0}^{t}\left\{U_{22}\left(r_{1}, r_{2}, r_{3}\right)\right. \\
\quad+\left[U_{2}\left(t^{\prime}\right)+U_{2}\left(t^{\prime \prime}\right)\right] U_{12}\left(r_{2}, r_{1}, r_{3}\right) \\
\left.\quad+U_{2}\left(t^{\prime}\right) U_{2}\left(t^{\prime \prime}\right) U_{11}\left(r_{2}, r_{1}, r_{3}\right)\right\} d t^{\prime} d t^{\prime \prime}
\end{aligned}
$$

where $r_{1}=t^{\prime}-t^{\prime \prime}, r_{2}=2 \beta \sin \left[\left(t^{\prime}-t^{\prime \prime}\right) / 2 \lambda\right] \cos \left[\left(t^{\prime}+t^{\prime \prime}\right) / 2 \lambda\right]$, $r_{3}=0$.

The difference between (19) and (23) is that we have replaced $v_{t J, 11}$ by the components of $U_{\imath \jmath}$, the velocity covariances for steady flow of unit mean velocity parallel to $x_{1}$ (A4). These components are given in (11) and were derived in the past [Rubin, 1990; Rubin and Dagan, 1992; Zhang and Neuman, 1992]. The evaluation of the contribution of each of the three terms of (23) showed that the midterm is responsible mainly for the small oscillatory part of $X_{11, I I} \approx X_{22}$ (Figures 5 and 8) and $D_{22}$ (Figures $7 \mathrm{c}$ and 9 ). The linear trend of the increment $X_{22}(t)-X_{22}^{(s t)}(t)$ (Figure 6, equations (21) and (22)) is associated with the remaining two terms, leading to the following approximate expressions for $X_{22}$ and $D_{22}$ :

$$
\begin{aligned}
X_{22}(t) & \approx \int_{0}^{t} \int_{0}^{t}\left[U_{22}\left(r_{1}, r_{2}, r_{3}\right)\right. \\
& \left.+U_{2}\left(t^{\prime}\right) U_{2}\left(t^{\prime \prime}\right) U_{11}\left(r_{2}, r_{1}, r_{3}\right)\right] d t^{\prime} d t
\end{aligned}
$$

with $r_{1}=t^{\prime}-t^{\prime \prime}, r_{2}=2 \beta \sin \left[\left(t^{\prime}-t^{\prime \prime}\right) / 2 \lambda\right) \cos \left[\left(t^{\prime}+t^{\prime}\right) / 2 \lambda\right]$, and $r_{3}=0$; and

$$
D_{22}(t) \approx \int_{0}^{t}\left[U_{22}\left(r_{1}, r_{2}, r_{3}\right)+U_{2}(t) U_{2}\left(t^{\prime}\right) U_{11}\left(r_{2}, r_{1}, r_{3}\right)\right\} d t^{\prime}
$$

with $r_{1}=t-t^{\prime}, r_{2}=2 \beta \sin \left[\left(t-t^{\prime}\right) / 2 \lambda\right) \cos \left[\left(t+t^{\prime}\right) / 2 \lambda\right]$, and $r_{3}=0$.
In the steady state case, i.e., for $\beta=0$, the only term left in the integrands of (24) is $U_{22}\left(t^{\prime}-t^{\prime \prime}, 0,0\right)$ along the $x_{1}$ axis, leading to the expressions derived in the past [e.g., Dagan, 1984]. Since $U_{22}\left(r_{1}, 0,0\right)$ is a hole covariance, $D_{22}^{(s t)}$ tends to zero for $t \gg 1$ in both two- and three-dimensional transport, while $X_{22}^{(s t)} \sim \ln t$ (two dimensions) and $X_{22}^{(s t)} \rightarrow$ const (three dimensions). Hence the linear trend present in $X_{22}$ or the constant, Fickian, contribution $\Delta \alpha_{T}$ (equations (21) and (22)) to $D_{22}$ originates from two terms in (24): The first one is $U_{22}$, the transverse velocity covariance integrated along the actual trajectory rather than the $x_{1}$ axis, and the second one is from the longitudinal velocity covariance $U_{11}$, however for the cross flow of mean velocity $U_{2}$ and with $r_{2}$ and $r_{1}$ exchanged in the argument of $U_{11}$. These terms are similar, but more complex, than those accounted for by Goode and Konikow [1990] (see section 1). It is recalled that $X_{22}$ and $D_{22}$ are proportional to $\sigma_{Y}^{2}$ and depend here in a complex manner on $\beta$ and $\lambda$.

The half slopes $\Delta \alpha_{T}$ of the linear trend of the increment $X_{22}(t)-X_{22}^{(s t)}$ incorporate in a single parameter the main unsteady effect investigated here. First, it leads to the simple approximation

$$
X_{22}(t ; \beta, \lambda) \approx X_{22}^{(s t)}(t)+2 \Delta \alpha_{T}(\beta, \lambda) t
$$

which neglects the oscillatory part of $X_{22}$, seen to be negligible in Figure 6.

By the same token, $D_{22}$ can be approximated by

$$
D_{22}(t ; \beta, \lambda) \approx D_{22}^{(s)}(t)+\Delta \alpha_{T}(\beta, \lambda)
$$

and we have plotted (26) in both Figures $7 \mathrm{c}$ and 9. It is seen that indeed $D_{22}$ in (26) averages the time oscillations of $D_{22}$, which are more pronounced than those of $X_{22}$.

The most important point, however, is that $\Delta \alpha_{T}$ in (25) and (26) represents a Fickian effect which becomes the dominant one for sufficiently large $t$, especially in three-dimensional flow. As a matter of fact, it is made dimensionless with respect to $U_{1} I_{Y}$, and therefore, $\Delta \alpha_{T}$ may be viewed as the ratio between the asymptotic macrodispersivity and the log conductivity integral scale. Its numerical values (equations (21) and (22)) suggest an approximate quadratic dependence upon $\beta$ and a decay with $\lambda(21)$.

The approximate results (equations (23) and (24)) will serve in the future for analysis of field tests in which $C_{Y}$ is generally of an anisotropic structure which does not lend itself to simple, analytical, expressions for the functions $P$ and $Q$ (equations (A2) and (A3)).

\section{Summary and Conclusions}

By using the Lagrangian approach and a first-order approximation of flow and transport equations, we have been able to derive the solute particles trajectory covariances $X_{i j}$ for an average mean flow uniform in space but varying in time. Under ergodic conditions that apply for sufficiently large plumes compared to heterogeneity scales, the trajectory covariances are equal to the plume spatial moments, while the centroid moves along the mean trajectory. In a similar vein, the $D_{i j}=$ $(1 / 2) d X_{\imath \jmath} / d t$ represent the effective dispersion coefficients of the plume.

Similarly to the steady flows investigated in the past, the $X_{i j}$ result from the integration of the velocity covariances $u_{t j}$ along the mean trajectory $\langle\mathbf{X}\rangle$, which departs from a straight line and depends nonlinearly on time. However, $u_{t}$ are the same as for 
steady state flow provided that the mean velocity is the instantaneous velocity of the actual flow.

We have illustrated the procedure by applying the general method to the particular case of an isotropic exponential log conductivity covariance and a periodic time-varying flow. Thus the mean velocity component $U_{1}$ is constant while the transverse $U_{2}$ varies harmonically in time.

Our main result was that for the selected values of parameters, the longitudinal dispersion of the plume is little affected by the unsteady flow. The plume rotates by the angle of the mean flow at $t=0$, and it becomes practically aligned with $x_{1}$ after a period.

The major impact of the unsteady component is on transverse dispersion. The effective dispersion coefficient $D_{\mathrm{II}, \mathrm{II}} \approx$ $D_{22}$ can be viewed as made up from three terms: (1) the steady state component, which results from the integration of the transverse velocity covariance $U_{22}$ along the $x_{1}$ axis and which tends to zero for large travel time; (2) a small periodic component stemming mainly from $U_{12}$, and (3) a constant, Fickian, additional term. The last term results from the integration of the transverse velocity covariance $U_{22}$ along the sinusoidal trajectory and from that of $U_{11}$ in the $x_{2}$ direction. The third category of terms encapsulates the main contribution of flow unsteadiness upon transport. These terms depend in a complex manner upon heterogeneity, being proportional to $\sigma_{Y}^{2}$, and on the amplitude and period of the time-varying $U_{2}$. This result is different from that of previous studies, in which heterogeneity and time dependence of the mean flow effects were separated.

\section{Appendix: A Few Analytical Auxiliary Results}

The fundamental functions $P(\mathbf{r})$ and $Q(\mathbf{r})$ [see, e.g., Dagan, 1989; Rubin, 1990] satisfy the equations

$$
\nabla^{2} P=-C_{r}(\mathbf{r}) \quad \nabla^{2} Q=P
$$

For an isotropic $C_{Y}$, (A1) become ordinary differential equations, with $\nabla^{2} \equiv(1 / r) d / d r(r d / d r)$ (two dimensions) and $\nabla^{2} \equiv\left(1 / r^{2}\right) d / d r\left(r^{2} d / d r\right)$ (three dimensions). For ease of reference we give here the final results for $C_{Y}=\sigma_{Y}^{2} \exp (-r)$, where $r$ is made dimensionless with respect to $I_{Y}$ :

Two dimensions

$$
\begin{gathered}
P /\left(I_{Y}^{2} \sigma_{Y}^{2}\right)=1-e^{-r}-E+\operatorname{Ei}(-r)-\ln (r) \\
Q /\left(I_{Y}^{4} \sigma_{Y}^{2}\right)=\frac{(r-5) e^{-r}}{4}+\frac{r^{2}}{2}\left(1-\frac{E}{2}\right)+\left(\frac{3}{2}+\frac{r^{2}}{4}\right) \operatorname{Ei}(-r) \\
-\frac{r+6}{4} \ln (r)
\end{gathered}
$$

Three dimensions

$$
\begin{gathered}
P /\left(I_{Y}^{2} \sigma_{Y}^{2}\right)=-1+\frac{2}{r}-e^{-r}-\frac{2 e^{-r}}{r} \\
Q /\left(I_{Y}^{4} \sigma_{Y}^{2}\right)=-3-e^{-r}+\frac{4}{r}-\frac{4 e^{-r}}{r}+r-\frac{r^{2}}{6}
\end{gathered}
$$

where $E$ is the Euler constant and $\mathrm{Ei}$ is the exponential integral. It is recalled that the velocity covariances (equations (10) and (11)) are expressed with the aid of the derivatives of $P$ and $Q$.

Next, we present the relationships between the tensor $v_{t, \alpha \beta}\left(r_{1}, r_{2}\right)$ (the covariance between the $i$ th component of the velocity fluctuation at $x$ caused by a unit mean flow in the $\alpha$ direction and the $j$ th fluctuation at $\mathbf{y}=\mathbf{x}-\mathbf{r}$ for a unit mean flow in the $\beta$ direction) as given by (10) and $U_{i j}\left(r_{1}, r_{2}\right)=$ $v_{t, 11}$ (equation (11)) for two-dimensional flow. Thus

$$
\begin{gathered}
v_{11,11}=U_{11} \quad v_{22,22}=U_{11}^{*} \\
v_{12,11}=v_{21,11}=v_{11,21}=v_{11,12}=U_{12} \\
v_{12,22}=v_{21,22}=v_{22,12}=v_{22,21}=U_{12}^{*} \\
v_{22,11}=v_{11,22}=v_{12,21}=v_{21,21}=U_{22}
\end{gathered}
$$

where $U_{i j}^{*}\left(r_{1}, r_{2}\right)=U_{i j}\left(r_{2}, r_{1}\right)$. The relationships are the same for three-dimensional flow with additional dependence on $r_{3}$.

Acknowledgment. This study was supported through NSF grant 9304481.

\section{References}

Dagan, G., Solute transport in heterogeneous porous formations, $J$. Fluid Mech., 145, 151-177, 1984.

Dagan, G., Flow and Transport in Porous Formations, 465 pp., Springer-Verlag, New York, 1989.

Dagan, G., Transport in heterogeneous formations: Spatial moments, ergodicity, and effective dispersion, Water Resour. Res., 26, 1281$1290,1990$.

Dagan, G., An exact nonlinear correction to transverse macrodispersivity for transport in heterogeneous formations, Water Resour. Res., 30, 2699-2705, 1994.

Farell, D. A., A. D. Woodbury, E. A. Sudicky, and M. O. Rivett, Stochastic and deterministic analysis of dispersion in unsteady flow at the Borden tracer-test site, Ontario, Canada, J. Contam. Hydrol., 15, 159-185, 1994.

Goode, D. J., and L. F. Konikow, Apparent dispersion in transient groundwater flow, Water Resour. Res., 26, 2339-2351, 1990.

Kabala, Z. J., and G. Sposito, A stochastic model of reactive solute transport with time-varying velocity in a heterogeneous aquifer, $\mathrm{Wa}$ ter Resour. Res., 27, 341-350, 1991.

Kinzelbach, W., and P. Ackerer, Modelisation de la propagation d'un contaminant dans un champ d'écoulement transitoire, Hydrogeologie, 2, 197-205, 1986.

Naff, R. L., T.-C. J. Yeh, and M. W. Kemblowski, A note on the recent natural gradient experiment at the Borden site, Water Resour. Res., 24, 2099-2104, 1988.

Neuman, S. P., and Y.-K. Zhang, A quasi-linear theory of non-Fickian and Fickian subsurface dispersion, 1, Theoretical analysis with application to isotropic media, Water Resour. Res., 26, 867-902, 1990.

Rehfeldt, K. R., and L. W. Gelhar, Stochastic analysis of dispersion in unsteady flow in heterogeneous aquifers, Water Resour. Res., 28, 2085-2099, 1992.

Rubin, Y., Stochastic modeling of macrodispersion in heterogeneous porous media, Water Resour. Res., 26, 133-142, 1990.

Rubin, Y., and G. Dagan, A note on head and velocity covariances in three-dimensional flow through heterogeneous anisotropic porous media, Water Resour. Res., 28, 1463-1470, 1992.

Sudicky, E. A., A natural-gradient experiment on solute transport in a sand aquifer: Spatial variability of hydraulic conductivity and its role in the dispersion process, Water Resour. Res., 22, 2069-2082, 1986.

Zhang, D., and S. P. Neuman, Comment on " $A$ note on head and velocity covariances in three-dimensional flow through heterogeneous anisotropic porous media" by Y. Rubin and G. Dagan, Water Resour. Res., 28, 3343-3344, 1992.

Zhang, Y.-K., and S. P. Neuman, A quasi-linear theory of non-Fickian and Fickian subsurface dispersion, 2, Application to anisotropic media and the Borden site, Water Resour. Res., 26, 903-914, 1990.

A. Bellin, Dipartamento di Ingegneria Civile ed Ambientale, Università di Trento, via Mesiano di Povo, 77, I-38050, Trento, Italy. (e-mail: alberto@itncal.ing.unitn.it)

G. Dagan, Faculty of Engineering, Tel Aviv University, P. O. Box 39040, Ramat Aviv 69978, Israel. (e-mail: dagan@eng.tau.ac.il)

Y. Rubin, Department of Civil Engineering, University of California, Berkeley, CA 94720.

(Received December 5, 1994; revised August 14, 1995; accepted August 17, 1995.) 